\title{
Diabetic Mastopathy Simulating Breast Cancer: A Case Report with Review of the Literature

\author{
F. Z. Abakka ${ }^{1 *}$, I. Zouita ${ }^{1}$, S. Kirami ${ }^{1}$, D. Basraoui ${ }^{1}$, H. Jalal ${ }^{1}$
}

${ }^{1}$ Radiology Department, Mother and Child Hospital, CHU Mohammed VI, Marrakech, Faculty of Medicine and Pharmacy of Marrakech, Morocco

DOI: $10.36347 /$ sjmcr.2021.v09i04.040

| Received: 17.03.2021 | Accepted: 25.04.2021 | Published: 30.04.2021

*Corresponding author: F. Z. Abakka

\section{Abstract}

Diabetic mastopathy (DM) is a pathological entity of recent description, it is rare and benign; occurring in young patients with type I diabetes, or with an autoimmune disease. Clinically, it simulates breast cancer. Complementary examinations are not very informative. The diagnosis of certainty is histological. Through an observation of DM in a diabetic patient, under insulin since the age of 10 years, we recall the clinical, radiological and evolutionary aspects of this affection.

Keywords: Mastopathy; Type 1 diabetes; Breast cancer.

Copyright $(\mathcal{C} 2021$ The Author(s): This is an open-access article distributed under the terms of the Creative Commons Attribution 4.0 International License (CC BY-NC 4.0) which permits unrestricted use, distribution, and reproduction in any medium for non-commercial use provided the original author and source are credited.

\section{INTRODUCTION}

Diabetes, which has developed over a long period of time, is the cause of various complications, affecting most organs. For a long time it was believed that the mammary gland was immune to these complications. It was not until 1984 that Soler and Khardori [1] described for the first time a case of breast lesions associated with long-standing diabetes. The term diabetic mastopathy (DM), commonly used, was introduced by Tomaszewski et al., [2]. This type of mastopathy is a rare, benign disease that occurs particularly in patients with old type I diabetes. They take on the appearance of a tumoral lesion simulating breast cancer both clinically and radiologically. We report a new case of MD in the light of which we discuss the diagnostic and management problems of this condition.

\section{Case Report}

The patient was 35 years old, married, with no family history of breast cancer, and had been followed since the age of 10 years for type I diabetes on insulin therapy. Her diabetes is well balanced, no degenerative complications have been detected.

She came to our clinic with a recent (2 months) nodule of the left breast. On inspection, two symmetrical breasts were found with no morphological abnormalities, in particular no nipple retraction, orange peel appearance or skin redness. On palpation, there was a poorly defined nodular formation $4 \mathrm{~cm}$ in diameter, of stony consistency, painless, mobile in relation to the superficial and deep planes and located at the union of the right upper quadrants. There was no breast discharge or axillary adenopathy. The examination of the left breast was normal.

The breast ultrasound (Fig-1) showed a hypoechoic area with very marked ultrasound attenuation and irregular contours that was highly suspicious.

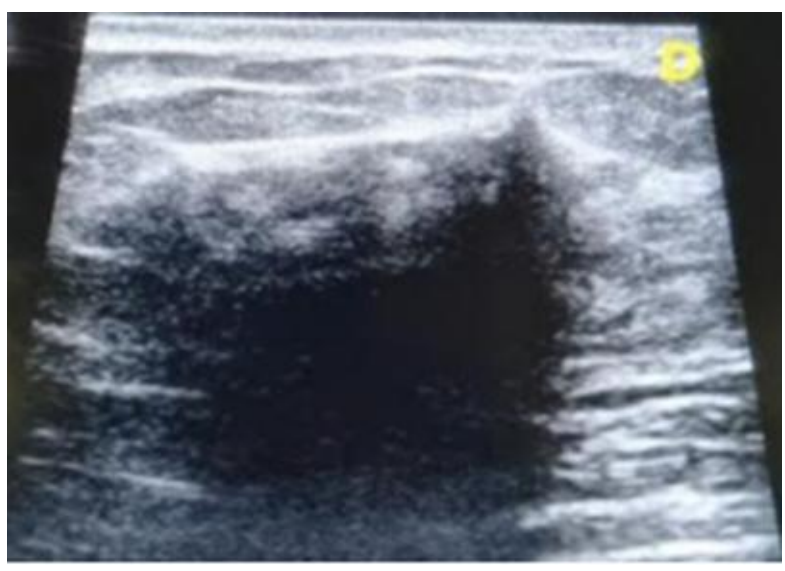


Fig-1: Breast ultrasound. Very hypoechoic absorbent area, with irregular and indistinct contours more marked in the right upper outer quadrant
Mammography (Fig-2) described dense breasts (Birads' D type), without architectural distortion or microcalcification.
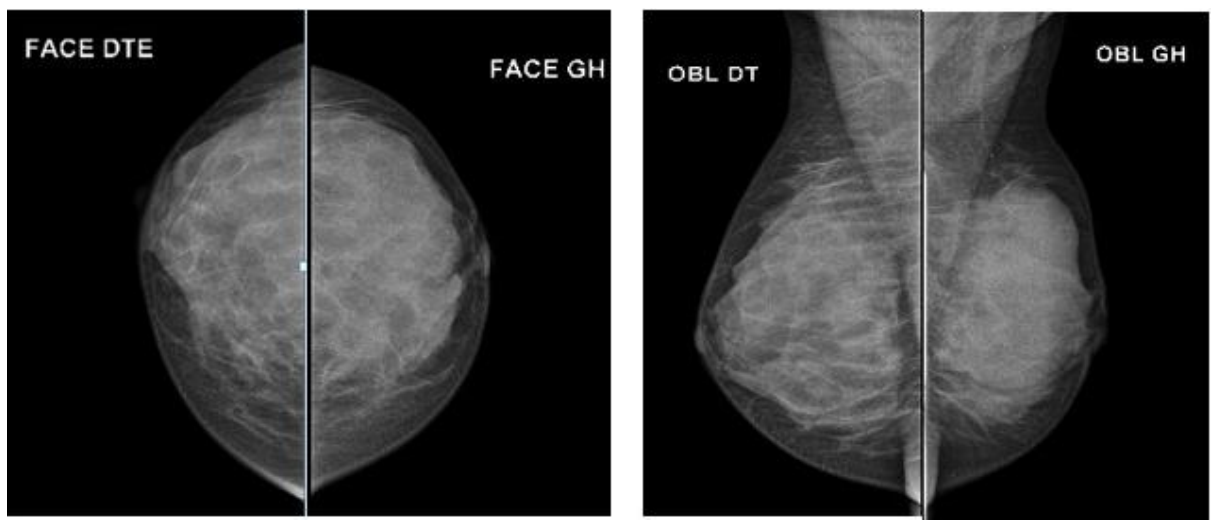

Fig-2: Mammography: dense breasts BIRADS type IV, without microcalcifications and without architectural distorsion

Breast MRI (Fig-3) showed bilateral micro nodular matrix enhancement type 3.
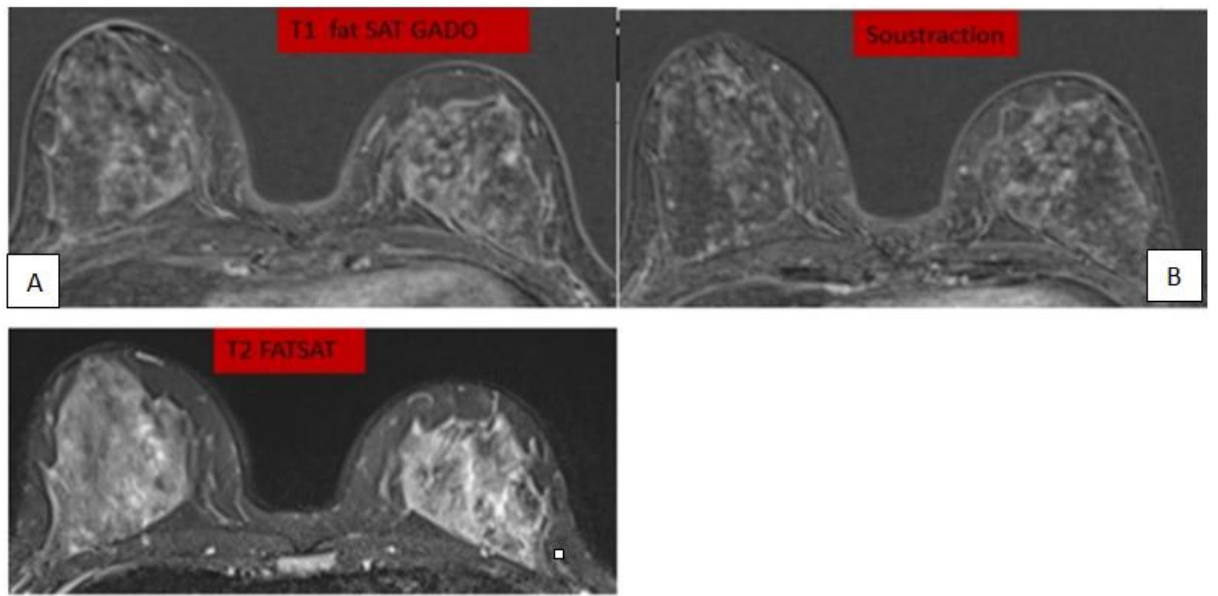

Fig-3: Breast MRI. T1 gadolinium axial sections (A) and T1 Fat Sat subtraction (B). Bilateral type 3 micro nodular matrix enhancement

A microbiopsy of the stony mass under ultrasound control was performed and the histological examination typically found a dense fibrous stroma, with a peri-lobular, peri-ductal and perivascular lymphocytic infiltrate. This lymphocytic infiltration was without signs of malignancy, suggesting a fibrous mastopathy of the breast.

\section{Discussion}

Diabetic mastopathy, also known as fibrous breast disease [1] or lymphocytic sclerosing lobulitis [3, 4], is a recent, rare and benign disease entity; about 200 cases have been reported in the literature. The prevalence of this disease is unknown due to the lack of knowledge of the condition and the fact that the reported publications have only concerned sporadic cases. The frequency of occurrence of these DM is estimated at $0.06 \%$.
This condition mainly affects young women with type I diabetes, frequently at the stage of degenerative complications [5]. More rarely, the same anatomical lesions have been described in other autoimmune pathologies (thyroiditis, lupus) or in type II diabetes [6]. Bilaterality is found in about $63 \%$ of cases [7]. Male involvement is exceptional; 5 cases have been reported in diabetic men [5]. Our patient is a type I diabetic, with degenerative complications of osteoarticular type. The pathophysiology of DM is not fully understood.

Some authors suggest a possible toxicity to exogenous insulin. Seidman et al., [8] have reported cases of MD in type II diabetic patients. They incriminate exogenous insulin in the genesis of these DM through an inflammatory or immune reaction. Clinically, DM always presents as a nodule of the breast, single or multiple, uni or bilateral, of recent onset, irregular in outline, hard or even stony in consistency, painless, often growing rapidly, mobile in 
relation to both the superficial and deep planes, variable in location, but frequently retroareolar, and measuring from 0.5 to $6 \mathrm{~cm}$ in long axis. It is not associated with nipple discharge or inflammatory skin phenomena. Our patient had a single nodule, poorly limited, of stony consistency, with a neoplastic appearance. These lesions clinically simulate breast cancer and it is difficult to distinguish these two entities by simple physical examination. For this reason, the use of complementary examinations or even surgery is essential. Radiological examinations provide little information; thus, mammograms frequently show overdensity, more or less regular opacities, or even dense breasts without architectural distortion or microcalcifications [6]. These ultrasound anomalies were found in our patient. Breast MRI has been tested in limited cases; however, it does not differentiate MD from possible breast cancer [9]. According to the majority of studies consulted, iconographic examinations lack sensitivity and specificity.

The aspects found resemble those of squirrel tumors and infiltrating lobular carcinomas; therefore, histological examinations are always essential for a definitive diagnosis.

Biopsy is therefore the examination of choice for diagnosis. The histological study reveals three kinds of elementary lesions, non-specific and variously associated: lymphocytic lobulitis, fibrosis of the mantle tissue, and inconstantly, homogeneously distributed stromal epithelioid cells.

When the diagnosis is made by micro biopsy radical surgery is not necessary. Simple surveillance is recommended, including an annual clinical examination and a mammogram every two years, it is possible that MD affects a healing process. No direct relationship between MD and breast cancer has been reported in the literature, and the risk of developing breast cancer later is similar to that of the general population [3].

\section{CONCLUSION}

Diabetic mastopathy is a rare and benign condition occurring mainly in young type I diabetic patients with multiple degenerative complications. It clinically simulates breast cancer. Mammography is not very informative and the diagnosis of certainty is based on histological examination. Its evolution is dominated by the risk of recurrence. After exclusion of a neoplastic pathology by micro biopsy, its management is limited to simple surveillance.

Conflicts of Interest: The authors declare no conflict of interest.

Authors' Contributions: All authors have read and approved the final version of the manuscript.

\section{REFERENCES}

1. Soler NG, Khardori R. Fibrous disease of the breast, thyroiditis and cheiroarthropathy in type 1 diabetes mellitus. Lancet. 1984;1:193-5.

2. Tomaszewski JE, Brouks JS, Hicks D, Li Volsi VA. Diabetic mastopathy: a distinctive clinicopathologic entity. Hum Pathol. 1992;23:7806.

3. Bouté V, Denoux Y, Feilel V, Lemery S, Lacroix J, Peffault De Latour M. Diabetic mastopathy: an uncommon complication of type I diabetes. J Le Sein. 2003;13:261-9.

4. Williams PH, Rubin CM, Theaker JM. Sclerosing lumphocyticlobulitis of the breast. Clin Radiol. 1995;50:165-7.

5. Kudva YC, Reynolds CA, O'Brien T, Crotty TB. Mastopathy and diabetes. Curr Diab Rep. 2003;3:56-9.

6. Hadj Ali I, Ben Abdallah N, Khiari K, Ben Salem L, Cherif L, Mrad K. Diabetic fibrous mastopathy about a case. Ann Endocrinol. 2002;63:235-9.

7. Murakami R, Kumita SI, Yamaguchi K, Ueda T. Diadetic mastopathy mimicking breast cancer. Clin Imaging. 2009;33:234-6.

8. Seidman JD, Schnaper LA, Phillips LE. Mastopathy in insulin-requiring diabetes mellitus. Hum Pathol. 1994;25:819-24.

9. Andrews-Tang D, Diamond AB, Rogers L, Butler D. Diabetic mastopathy: adjunctive use of ultrasound and utility of core biopsy in diagnosis. Breast J. 2000;6:183-8. 